\title{
Estudio de discursos políticos y mediáticos en las elecciones gallegas de 2020
}

\section{A study on the political and media discourses in the 2020 Galician regional elections}

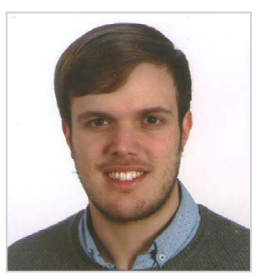

Recibido: 23/12/2020 - Aceptado: 27/09/2021 - En edición: 14/10/2021 - Publicado: 01/12/2021 Resumen:

La crisis sanitaria del Covid-19 transformó los patrones de consumo informativo y modificó el contexto comunicativo de procesos electorales previstos para 2020. Así sucedió con las elecciones autonómicas gallegas, que desplazaron su celebración de abril a julio de ese año. Ante esta circunstancia excepcional, resulta de interés explorar el desarrollo de esa campaña electoral, singularmente a través de los temas que articularon la agenda tanto para los candidatos como para los medios de comunicación. La estabilidad del sistema electoral gallego puede conllevar que solo fenómenos imprevistos como la pandemia de coronavirus provoquen disrupciones en el voto. Este estudio analiza la configuración temática de la campaña a las elecciones gallegas de 2020. Se examina tanto la agenda política, construida a partir de los mensajes en Twitter y el debate electoral televisado, como la agenda mediática que exponen los principales periódicos de la comunidad autónoma. Se profundiza sobre hasta qué punto el Covid-19 domina la campaña y si se produce una asimilación entre los asuntos priorizados por los medios y los candidatos, observando además posibles diferencias entre Twitter y la televisión. Los resultados revelan una agenda fragmentada, sin que se correlacione el contenido de los diversos formatos.

\section{Palabras clave:}

Comunicación política; agenda política; agenda mediática; elecciones gallegas; Covid-19.
Received: 23/12/2020 - Accepted: 27/09/2021 - Early access: 14/10/2021 - Published: 01/12/2021

\section{Abstract:}

The covid-19 pandemic has changed not only news consumption but also the communication in electoral processes due to take place in the Spring of 2020. This is the case of the Galician regional elections, which were postponed from April to July of that year. Therefore, in the context of a pandemic, it is of interest to explore the thematic agenda of that electoral campaign, analysing the topics preferred by the candidates and the media on several platforms. The stability of the voting intention in Galicia may mean that only singular phenomena such as the coronavirus outbreak would disrupt its political system. This research analyses the thematic distribution of the messages in the Galician election campaigns 2020, assessing the political agenda on Twitter and the TV electoral debate and the media agenda by studying the leading newspapers in the region. It delves into the extent to which Covid-19 focuses on communication, gauging whether issues emerge and whether the media and the candidates prioritise the same issues. The results show a fragmented agenda, with no correlation between the content of the various formats.

\section{Keywords:}

Political communication; political agenda; media agenda; Galician regional election; COVID-19.

Cómo citar este artículo:

Rivas-de-Roca, R. (2021). Estudio de discursos políticos y mediáticos en las elecciones gallegas de 2020. Doxa Comunicación, 33, pp. $121-136$. https://doi.org/10.31921/doxacom.n33a1492 


\section{Introducción}

Las elecciones son un momento de fuerte carga simbólica y comunicativa. En 2020, la aparición del Covid-19 alteró los marcos de los agentes implicados en la comunicación política, afectando especialmente a las elecciones autonómicas (Euskadi y Galicia) programadas para la primavera de ese año. La pandemia supuso un cambio en el consumo informativo de los ciudadanos, que se orientaron hacia una mayor polarización (Masip et al., 2020). No obstante, los primeros estudios muestran que los medios convencionales se erigieron como fuente informativa fiable (Aleixandre-Benavent et al., 2020; Casero-Ripollés, 2020), y que reforzaron su función social en un momento de distanciamiento social.

La literatura subraya que la comunicación política actual viene marcada por su naturaleza híbrida, que aúna la lógica mediática tradicional con las tecnologías digitales (Vaccari et al., 2015). Este doble proceso afecta a partidos políticos y movimientos sociales (Labio y Pineda, 2016), pero no impide que redes sociales como Twitter funcionen como un espacio clave para las campañas electorales (López Meri, 2016).

En este marco híbrido y a la vez fuertemente digitalizado, la celebración de las elecciones gallegas se pospuso al 12 de julio de 2020. El contexto comunicativo fue distinto debido a la pandemia, lo que justifica que esta investigación analice las prioridades temáticas de los candidatos (agenda política) y de los medios (agenda mediática). El objetivo es conocer a grandes rasgos la configuración de la campaña electoral.

Galicia constituye un caso de interés, donde el Partido Popular (PPdG) es desde hace años la fuerza hegemónica. De hecho, solo en dos ocasiones desde 1981 no ha obtenido mayoría absoluta. Esto puede ocasionar que solo eventos singulares impacten en su sistema político. Las elecciones gallegas celebradas hasta la fecha han estado marcadas por un escaso peso del nacionalismo, que se contrapone a una visión regionalista transversal (Gómez-Reino, 2009). El estudio de las agendas, entendidas como los asuntos que ocupan a la opinión pública, los medios o el público (Humanes y Moreno, 2012; Ardévol-Abreu et al., 2020), supone un tema apenas abordado en los comicios autonómicos (Fenoll et al., 2016; Gamir Ríos, 2016).

Teniendo en cuenta lo anterior, esta investigación pretende profundizar en la composición temática de la campaña electoral para las elecciones gallegas. Para ello, se efectúa un análisis de la agenda política, desagregada en el debate electoral de la Televisión de Galicia y los mensajes difundidos en Twitter, mientras que la agenda mediática se valora a través de los contenidos de campaña publicados por la prensa líder en la región.

Este estudio busca determinar la composición temática de la campaña para las elecciones gallegas de 2020. Para ello se procede a un análisis de la agenda política a través de los debates electorales televisados y los mensajes difundidos en Twitter, mientras que la agenda mediática se evalúa mediante los contenidos sobre la campaña publicados por los medios escritos líderes en la región. La interrelación entre formatos tradicionales y las redes sociales es habitual (Rúas-Araújo et al., 2020), así que el objetivo es valorar el grado de asimilación entre medios y candidatos durante el período electoral. 


\section{Estudio de la comunicación política y las agendas}

\subsection{Twitter y la comunicación política en campaña}

La convivencia de políticos y periodistas se basa en la influencia mutua, en la que tratan de intervenir sobre el otro con la meta de condicionar la opinión pública (Brants et al., 2009). Estos dos sujetos conforman junto con la ciudadanía los tres elementos fundamentales de la comunicación política (Mazzoleni, 2010). Las campañas electorales suponen momentos de especial intensidad que se insertan en la comunicación política. En ellas, los candidatos elaboran una determinada agenda para conseguir votos.

Las campañas contemporáneas desarrollan gran parte de sus acciones en internet (Stromer-Galley, 2014). La televisión como producto de masas en el siglo XX, unido a la proliferación de las tecnologías digitales en el siglo XXI, han generado una mediatización de la política. Por tanto, la comprensión de los asuntos públicos está asociada a la imagen proporcionada por los medios de comunicación (Strömbäck, 2008) y, en la actualidad, por la Red (Pérez-Curiel et al., 2021).

El desarrollo tecnológico implica otras consecuencias como la personalización de la política (McAllister, 2007; Rodríguez-Virgili et al., 2011), que espolea una preponderancia de los liderazgos individuales, y la espectacularización de la información televisiva (Berrocal, 2011). Esta tendencia al infoentretenimiento afecta a los debates electorales, en tanto que los candidatos prefieren mensajes efectistas por encima de propuestas programáticas (Wagner, 2016).

Las redes sociales, y en particular Twitter por su adaptación a la inmediatez informativa, han causado una revolución en la comunicación política (Parmelee y Bichard, 2012; Jungherr, 2016). En el caso de los períodos electorales, Twitter sirve tanto para candidatos como para periodistas y electores (Campos-Domínguez, 2017). Esta importancia creciente de las herramientas digitales conduce a una profesionalización de los partidos políticos, en el sentido de desarrollar una estrategia comunicativa ceñida a objetivos definidos (Tenscher et al., 2016). Destaca su carácter autorreferencial, ya que las propias actividades de los partidos y candidatos funcionan como temas principales en Twitter (Zugasti Azagra y García Ortega, 2018).

En un contexto de limitación del contacto social impuesto por la pandemia, el uso de plataformas digitales puede cobrar aún más importancia. Hasta ahora, las investigaciones sobre elecciones autonómicas han hallado que los candidatos emplean Twitter de una manera unidireccional muy similar a la efectuada por los medios tradicionales (Cebrián et al., 2013; Deltell et al., 2013). Sin embargo, emergen notables divergencias en función de factores como la trayectoria del partido o su posición en el eje gobierno-oposición (López-Meri et al., 2017). La atención académica sobre Twitter también se dirige a etapas poselectorales (Casero-Ripollés et al., 2021), en las que se debate la formación de un nuevo gobierno. Las graves consecuencias sanitarias y económicas del Covid-19 convierten al tiempo de la pandemia en un excelente campo de pruebas para conocer el uso de esta plataforma.

\subsection{Agendas políticas y mediáticas en España}

La teoría de la agenda-setting (McCombs y Shaw, 1972) constituye una influyente hipótesis en el estudio de los contenidos políticos de campaña, que goza de una amplia trayectoria de aplicación científica en España (Ardévol-Abreu et al., 2020). De acuerdo con la aportación inicial de esta teoría, los medios poseen la capacidad de transferir relevancia a ciertos temas mediante su selección de la actualidad informativa. Sin embargo, estas cuestiones temáticas de interés no solo se definen en el ámbito periodís- 
tico, sino también por los otros dos actores del sistema democrático: la clase política (agenda política) y los ciudadanos (agenda personal) (Carballo et al., 2018).

La transferencia de relevancia se sitúa siempre dentro de un marco temporal (McCombs, 2006). Aunque existen pluralidad de agendas dentro de un mismo entorno político, las investigaciones de agenda-setting se han centrado en analizar las prioridades temáticas de los medios a través del análisis de contenido (Guo y McCombs, 2015). En consecuencia, la agenda política ha sido menos estudiada que los intereses mediáticos y ciudadanos (Castromil et al., 2020). La agenda política se antoja más difícil de objetivar, puesto que los temas principales de los partidos aparecen en una gran variedad de espacios, como sus intervenciones públicas, sus programas electorales o, más recientemente, la actividad en redes sociales (Kim et al., 2017).

El objetivo de todo candidato es que los temas centrales de la campaña pivoten sobre su agenda política, lo que significa haber orientado a la opinión pública hacia los postulados de su partido. Más allá de que existan numerosas variables que influyen en esta agenda, los mensajes políticos en Twitter (Campos-Domínguez, 2017) y la actuación en los debates electorales (García Marín, 2015) son fórmulas para entender su composición. Estos canales se hallan vinculados hoy en día, al emplear las propias corporaciones audiovisuales estrategias que maximizan el seguimiento en Twitter de los debates televisivos (Rúas-Araújo et al., 2020). En el caso de España, se observa una traslación directa entre el medio televisivo para informarse y la intención de voto (Chavero-Ramírez et al., 2013).

La polarización del sistema político-mediático español hace que la transferencia de los temas de campaña a la ciudadanía dependa de la experiencia vital del individuo, lo cual explica comportamientos de exposición selectiva en el consumo de información política (Humanes, 2014). Los partidos políticos han demostrado su capacidad de influir en la agenda de medios tradicionales y digitales durante procesos electorales en España (Valera Ordaz, 2015), pero en esta construcción de agenda (agenda-building) la clásica mediatización parece dar pie a una interdependencia entre los actores involucrados (López García et al., 2017).

La tematización de las campañas se vincula con la actualidad (Chavero-Ramírez et al., 2013). En este sentido, el Covid-19 marcó el interés informativo en 2020 (Aleixandre-Benavent et al., 2020), si bien los comicios regionales que tuvieron lugar durante ese año tienen una trayectoria política propia. Como se comentó anteriormente, la centralidad en el discurso es habitual en las elecciones gallegas (Ares y Rama, 2019), y ha motivado una posible priorización de temas al respecto. Hasta la fecha apenas se han producido estudios de agendas acerca del panorama autonómico español, pero no es impedimento para considerar investigaciones que señalan una creciente mediatización hacia Twitter en esos espacios geográficos (Gamir Ríos, 2016; López Meri, 2016).

\section{Metodología}

Esta investigación tiene como objetivo examinar la distribución temática de las agendas políticas y mediáticas para las elecciones al Parlamento de Galicia, celebradas el 12 de julio de 2020. Se emplea un análisis de contenido (Krippendorff, 2012), dirigido a tres manifestaciones discursivas: mensajes en Twitter de los candidatos, debate electoral televisivo y piezas periodísticas en los principales diarios. El estudio de los tuits y las referencias temáticas en el debate se operacionalizan para conocer la agenda política de los líderes que concurrieron a las elecciones, mientras que los contenidos informativos revelan la agenda mediática de la prensa. Este artículo tiene una finalidad exploratoria, de la que se derivan varios objetivos: 
O1. Conocer las temáticas presentadas en Twitter por parte de los candidatos a las elecciones gallegas de 2020.

O2. Valorar la atención generada por la acción de los líderes en Twitter, que se formula a través de retuits.

O3. Evaluar cuantitativamente los temas abordados por cada candidato en el debate televisivo.

O4. Analizar el tratamiento mediático de los comicios en la prensa, y profundizar en cuáles han sido los temas y partidos preferidos.

En relación a Twitter, se emplea un análisis de contenido específico para esta red social (Fernández Crespo, 2014). El tipo de muestreo es intencional, dirigiendo el estudio hacia los tres candidatos que obtuvieron representación parlamentaria en las elecciones gallegas de 2020: Alberto Núñez Feijóo (@FeijooGalicia) del PPdG, Ana Pontón (@anaponton) del BNG (Bloque Nacionalista Galego) y Gonzalo Caballero (@G_Caballero_M) del PSdG (Partido Socialista de Galicia). La razón para elegir únicamente esas cuentas radica además en que estos partidos son clásicos dentro de la comunidad autónoma (Gómez-Reino, 2009).

El corpus para Twitter se compone de todos los tweets propios de los candidatos durante la campaña electoral (26 de junio-10 de julio de 2020), así como los publicados en los tres días posteriores a los comicios (13, 14 y 15 de julio) para conocer el impacto de los resultados en los mensajes. La recogida de los datos de Twitter se efectuó mediante la aplicación de Twitonomy.

En las elecciones gallegas de 2020 solo hubo un debate electoral televisivo en el que participaran los líderes seleccionados: el retransmitido por la Televisión de Galicia el 29 de junio de ese año. Como forma de acceder a su agenda temática, se abogó por su visionado íntegro y contabilizar el tiempo (en segundos) que cada candidato dedicaba a los ítems. El volumen en segundos para cada líder es reducido, dado que hasta siete partidos participaron en ese debate.

En cuanto al análisis de los medios, el corpus se compone de todas las informaciones con contenidos políticos sobre las elecciones gallegas, publicadas en su edición impresa. La franja temporal es similar a la establecida para Twitter (26 de junio-10 de julio, 13, 14 y 15 de julio de 2020). Los dos diarios analizados son La Voz de Galicia, el Faro de Vigo, El Progreso de Lugo y El Correo Gallego, periódicos de referencia en diferentes localidades de la comunidad autónoma y que disponen además de hemeroteca accesible para el investigador. En lugar de llevar a cabo un peinado sistemático de los diarios, la recopilación de las piezas periodísticas se realiza a partir de una búsqueda de palabras clave como "elecciones" o "campaña" dentro del conjunto del periódico. De esta forma se asegura la objetividad de la muestra y se facilita su posterior recuperación.

Los datos de los tres objetos de estudio (Twitter, debate electoral y medios) se han recogido mediante una ficha de análisis homogénea sobre cuestiones temáticas, construida específicamente para este artículo siguiendo principios de la investigación mixta entre elementos digitales y tradicionales (Sádaba Rodríguez, 2012). El modelo de estudio se desarrolla por un único investigador, puesto que los elementos temáticos no implican tanta diferencia en su interpretación como otras variables. En cualquier caso, el autor efectuó dos rondas previas de entrenamiento para definir mejor las categorías excluyentes de la ficha.

A pesar de que cada medio (Twitter, televisión y prensa) posee sus propias particularidades, esta investigación asume que las agendas son intercambiables entre estos espacios. La recogida de datos arroja una cifra de unidades de análisis que asciende a 386 tuis, 296 piezas periodísticas y 150 minutos de debate electoral. La ficha de categorización presenta elementos temáticos que son fruto de un análisis temático previo de Twitter, y que sigue también postulados de Patterson (1980) para fijar cuáles eran los tópicos esperados durante la campaña. 


\section{Resultados}

\subsection{Agendas políticas y atención generada en Twitter}

En primer lugar, las agendas políticas se desgranan analizando los 148 tuits de Alberto Núñez Feijóo, 163 de Gonzalo Caballero y 75 de Ana Pontón. La distribución temática revela evidencias empíricas de interés, entre las que destaca una cierta fragmentación de la agenda (tabla 1). Frente a esta dinámica, Caballero (PSdG) y Pontón (BNG) presentan un menor número de tópicos. Esto significa una mayor concentración temática de los candidatos de la oposición. La líder nacionalista prioriza los eventos políticos (28\%), como los actos de campaña, y la idea de cambio de ciclo (26,7\%). Por su parte, el candidato socialista también ubica a los eventos como su cuestión primordial (35\%). En cambio, su segundo tema es la movilización del voto, para lo que solicita el apoyo de los electores.

Tabla 1. Distribución de los tuits de los candidatos según su temática (\%)

\begin{tabular}{|l|c|c|c|c|}
\hline & $\begin{array}{c}\text { Alberto } \\
\text { Núñez Feijóo }\end{array}$ & $\begin{array}{c}\text { Gonzalo } \\
\text { Caballero }\end{array}$ & $\begin{array}{c}\text { Ana } \\
\text { Pontón }\end{array}$ & Total \\
\hline Cambio político & 0 & 14,1 & $\mathbf{2 6 , 7}$ & 11,1 \\
\hline Movilización voto & 15,5 & $\mathbf{1 5 , 3}$ & 1,3 & $\mathbf{1 2 , 7}$ \\
\hline Estrategia poselectoral & 9,5 & 4,9 & 0 & 5,7 \\
\hline Eventos políticos & 10,8 & $\mathbf{3 5}$ & $\mathbf{2 8}$ & $\mathbf{2 4 , 4}$ \\
\hline Economía/industria & $\mathbf{2 1 , 6}$ & 6,7 & 1,3 & 11,4 \\
\hline Servicios públicos & $\mathbf{1 6 , 9}$ & 5,5 & 0 & 8,8 \\
\hline Covid-19 & 8,8 & 9,2 & 9,3 & 9,1 \\
\hline Galicia & 10,8 & 2,5 & 10,7 & 7,3 \\
\hline Otros & 6,1 & 6,7 & 22,6 & 9,6 \\
\hline
\end{tabular}

Fuente: elaboración propia (en negrita resultados de mayor interés)

En comparación con la oposición, la agenda de Núñez Feijóo versa sobre temas como la economía (imagen 1) o los servicios públicos, lo que encaja con la imagen de gestor que un presidente intenta mostrar. Asimismo, referencia de manera profusa la movilización del electorado, algo que puede encontrarse motivado por un contexto de pandemia propicio a la abstención. En el conjunto de la investigación en Twitter, los eventos políticos y la movilización son las cuestiones sobre las que ha girado la campaña digital de los principales líderes. 
Imagen 1. Ejemplos de tuits relativos a economía (Feijóo) y cambio político (Pontón)

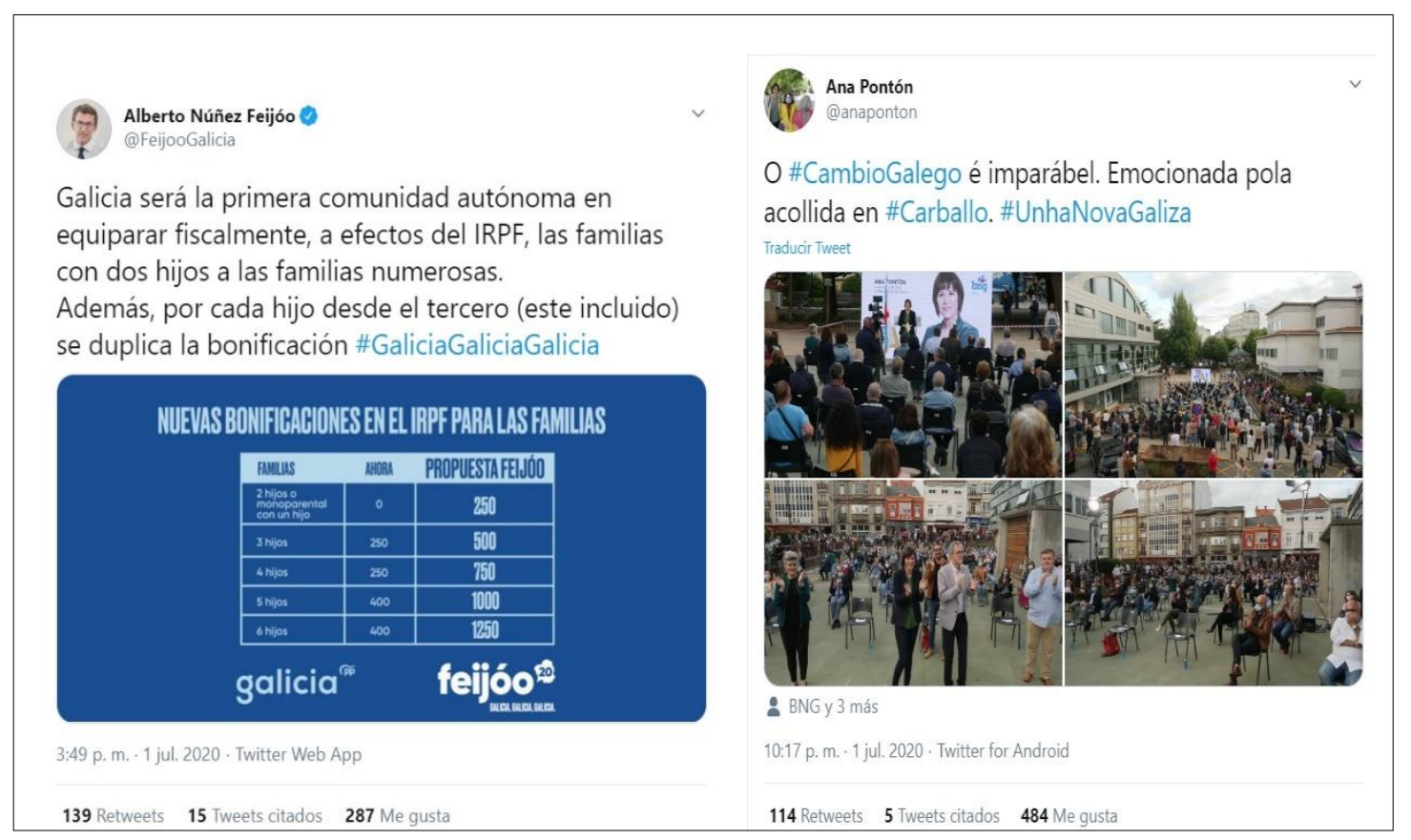

\section{Fuente: Twitter}

Para este estudio también es importante conocer el impacto cuantitativo en los usuarios de los mensajes de los candidatos en Twitter. Como se observa en la tabla 2, los resultados evidencian que los elementos temáticos que generan una mayor interacción (retuits) no coinciden con los más difundidos por los líderes políticos. Este hallazgo se halla en consonancia con lo apuntado por la literatura en materia de interacción con las estrategias de Twitter (Larsson e Ihlen, 2015; Rivas-de-Roca et al., 2021).

En la línea anterior, el tema "Covid-19" supone el 33,3\% de los mensajes por encima de 300 retuits y el 19,3\% entre 100 y 299 retuits, a pesar de que solo constituía el 9,1\% del total de la muestra. En cualquier caso, los siguientes ítems que causan más interacción (eventos políticos y movilización del voto) entroncan con la agenda construida por los líderes en Twitter, si bien "eventos" tiene su vez el 40,7\% de sus publicaciones en la franja con menor atención. 
Tabla 2. Grado de atención (media de retuits) en función de la temática (\%)

\begin{tabular}{|l|c|c|c|c|}
\hline & $\mathbf{1 - 4 9}$ & $\mathbf{5 0 - 9 9}$ & $\mathbf{1 0 0 - 2 9 9}$ & $\mathbf{3 0 0}$ o más \\
\hline Cambio político & 11,3 & 12 & 10,2 & 0 \\
\hline Movilización voto & 7,3 & 16,9 & $\mathbf{1 4 , 8}$ & 16,7 \\
\hline Estrategia poselectoral & 3,3 & 4,2 & 12,5 & 0 \\
\hline Eventos políticos & 40,7 & 15,5 & 10,2 & $\mathbf{3 3 , 3}$ \\
\hline Economía/industria & 6,7 & 20,4 & 5,7 & 0 \\
\hline Servicios públicos & 10 & 9,2 & 6,8 & 0 \\
\hline Covid-19 & 3,3 & 7,7 & $\mathbf{1 9 , 3}$ & $\mathbf{3 3 , 3}$ \\
\hline Galicia & 4 & 6,3 & 13,6 & 16,7 \\
\hline Otros & 13,3 & 7,7 & 6,7 & 0 \\
\hline
\end{tabular}

Fuente: elaboración propia (en negrita resultados de mayor interés)

La media de retuits permite estimar que el coronavirus ha conseguido alterar la organización temática de la campaña, al menos en lo referente a que el propio Covid-19 se erija en el tema que despierte una mayor atención en Twitter. Como se comentó antes, las elecciones no tuvieron lugar en un contexto normal, lo que obliga a tomar los resultados con cautela y llevar a cabo estudios comparados con comicios después de la pandemia.

En cuanto al único debate electoral con los tres candidatos aquí considerados, la distribución de los tópicos en segundos se refleja en el gráfico 1. Conviene señalar que se categorizan únicamente las intervenciones temáticas, dejando de lado las reacciones a los adversarios y las respuestas que no tienen que ver con temas estrictamente de políticas públicas o de campaña.

\section{Gráfico 1. Temas abordados por cada candidato en el debate televisivo (segundos)}

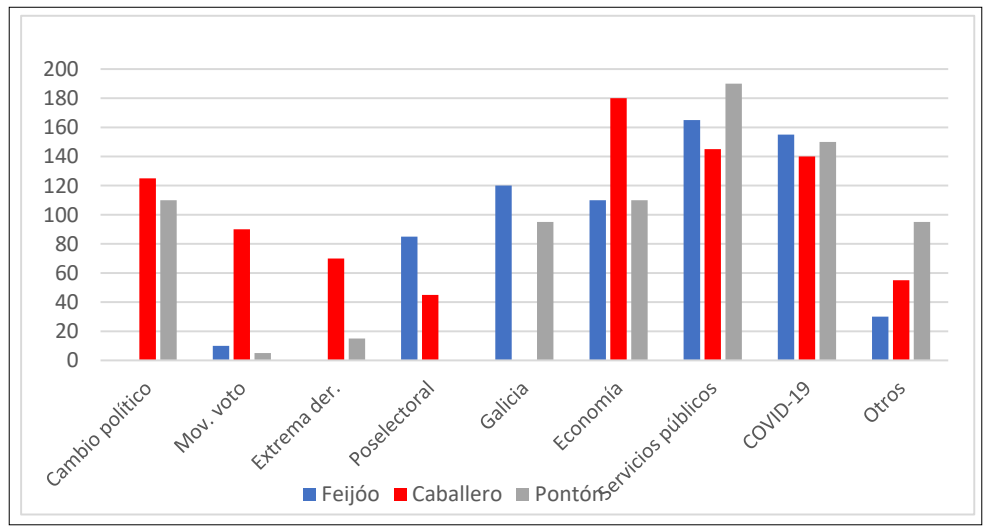

Fuente: elaboración propia 
Los partidos participantes acordaron con la Televisión de Galicia cuatro grandes bloques temáticos para el debate: Covid-19, economía, políticas sociales y gobernabilidad. Esta sujeción a cuestiones prefijadas supone una diferencia sustancial frente a Twitter, que puede explicar el hecho de que la agenda en el debate electoral resultara más programática. En el debate, los servicios públicos y la economía fueron temas muy referenciados por los tres candidatos.

Por su parte, destaca el elevado número de segundos dedicado al cambio político por los partidos de la oposición (PSdG y BNG), algo lógico en virtud de su objetivo de alcanzar la presidencia de la Xunta. Por su parte, Núñez Feijóo es el candidato que más referencia las estrategias postelectorales, criticando posibles pactos, y posiciona a Galicia como tema singular. Su lema de campaña fue "Galicia, Galicia, Galicia”.

Cuantitativamente, el candidato popular intervino menos en el debate, hasta un total de 140 segundos por debajo de sus dos principales contrincantes. La percepción de que la actuación televisiva influye sobre el voto (Lagares Díez et al., 2020) puede haber contribuido a esta posición más proactiva por parte de la oposición, mientras que la figura en el gobierno necesita menos tiempo para exponer sus planteamientos.

\subsection{Tratamiento informativo en la prensa de referencia}

Para conocer la agenda mediática de los comicios se procede a mostrar la distribución temática de las 296 piezas periodísticas analizadas, que corresponden a medios de referencia con la capacidad de marcar la discusión pública en Galicia (tabla 3). Se estudian 77 textos de La Voz de Galicia, 101 del Faro de Vigo, 66 de El Progreso de Lugo y 52 de El Correo Gallego.

Tabla 3. Distribución de las temáticas por periódico (\%)

\begin{tabular}{|c|c|c|c|c|c|}
\hline & $\begin{array}{c}\text { La Voz de } \\
\text { Galicia }\end{array}$ & Faro de Vigo & $\begin{array}{c}\text { El Progreso de } \\
\text { Lugo }\end{array}$ & $\begin{array}{c}\text { El Correo } \\
\text { Gallego }\end{array}$ & Total \\
\hline Cambio político & 7,8 & 14,9 & 6,1 & 7,7 & 9,8 \\
\hline Movilización voto & 19,5 & 10,9 & 7,6 & $\mathbf{2 8 , 8}$ & 15,5 \\
\hline Estrategia poselectoral & 26 & 15,8 & 28,8 & 25 & 23 \\
\hline Eventos políticos & 29,6 & 16,8 & 25,8 & 19,2 & 22,6 \\
\hline Economía/industria & 7,8 & 13,9 & 12,1 & 7,7 & 10,8 \\
\hline Servicios públicos & 3,9 & 16,8 & 4,5 & 0 & 7,8 \\
\hline Covid-19 & 5,2 & 4 & 10,6 & 9,6 & 6,8 \\
\hline Otros & 0 & 7 & 4,5 & 1,9 & 3,8 \\
\hline
\end{tabular}

Fuente: elaboración propia (en negrita resultados de mayor interés)

En el análisis de medios no se emplea la categoría “Galicia”, al ser una temática que en este caso se inserta en las demás. Los resultados indican una preferencia por los eventos políticos como tema noticioso, a semejanza de los retuits. En cambio, se difiere en la importancia de la estrategia postelectoral, que en los diarios escogidos supone un tema central para conocer los futuros pactos de gobierno, independientemente de la línea editorial. Además, se detecta que El Correo Gallego publica de manera notable so- 
bre movilización del voto, mientras que el Faro de Vigo lo hace acerca de servicios públicos. El diario olívico es el que expone una mayor pluralidad en los temas de sus piezas periodísticas.

Para esta investigación también se antoja útil detallar el volumen de referencias a los partidos en los diarios seleccionados. Según la tabla 4, se experimenta una preponderancia de los enfoques mixtos (35,1\%) y aquellos que se centran en el PPdG (27,7\%). En todos los periódicos se repite esta tendencia, si bien la presencia del Partido Popular de Galicia en El Correo Gallego es sumamente abundante. El sistema político de esta comunidad autónoma se caracteriza por la centralidad en torno a esa formación, que se refleja en su ubicación privilegiada en el ámbito mediático.

Tabla 4. Partido objeto de la información por cada diario (\%)

\begin{tabular}{|c|c|c|c|c|c|}
\hline & $\begin{array}{c}\text { La Voz de } \\
\text { Galicia }\end{array}$ & Faro de Vigo & $\begin{array}{c}\text { El Progreso de } \\
\text { Lugo }\end{array}$ & $\begin{array}{c}\text { El Correo } \\
\text { Gallego }\end{array}$ & Total \\
\hline PPdG & 22,1 & 26,7 & 24,2 & 42,3 & 27,7 \\
\hline PSdG & 19,5 & 25,7 & 21,2 & 21,2 & 22,3 \\
\hline BNG & 15,6 & 20,8 & 15,2 & 1,9 & 14,9 \\
\hline Mixto & 42,9 & 26,7 & 39,4 & 34,6 & 35,1 \\
\hline
\end{tabular}

Fuente: elaboración propia (en negrita resultados de mayor interés)

Por último, sobresale que el Faro de Vigo presente una cobertura relativamente homogénea en cuanto a número de referencias por partido. Todas las categorías superan el $20 \%$, en un hallazgo que no se produce en el resto de periódicos, que informan sensiblemente menos de los partidos de la oposición (PSdG y BNG). El nacionalista BNG apenas es referenciado en El Correo Gallego $(1,9 \%)$. El citado medio ignora así a un partido que fue la segunda opción más votada en las elecciones, poniendo en cuestión la representatividad del tratamiento periodístico efectuado.

\section{Discusión y conclusiones}

Como discusión, cabe resaltar que los comicios gallegos de 2020 contaron con muchas formaciones en liza que incluso fueron invitadas al debate televisivo (Ciudadanos, Vox, Galicia en Común-Anova Mareas y Marea Galeguista), pero solo los tres partidos tradicionales (PPdG, PSdG y BNG) obtuvieron diputados. Esta aparente vuelta a la "vieja política" imbrica con el hecho de que las agendas políticas en Twitter de los candidatos no parecieran haberse visto afectadas por el Covid-19. Núñez Feijóo se centra en asuntos que trasladan de él una imagen de buen gestor, como la economía y los servicios públicos, mientras que Caballero y Pontón aluden a la necesidad de cambio político. En este sentido, la campaña se desarrolló en parámetros clásicos, ajustados a un contexto fuertemente identitario en términos regionales (Warf y Ferras, 2015).

Frente al inmovilismo temático de los candidatos, los ciudadanos muestran un cambio en sus preferencias a raíz de la pandemia. De hecho, el Covid-19 es el asunto que genera más retuits. La agenda política en el debate electoral sí menciona de manera profusa al coronavirus, así como a los issues "servicios públicos” y “economía”, que constituían los temas de Núñez Feijóo en Twitter. Sería interesante reflexionar sobre si la organización del debate por parte de la televisión pública gallega -acusada de manipulación por el colectivo Defende a Galega- pudo beneficiar al actual presidente de la Xunta, al ajustarse a determinados bloques. 
Asimismo, la agenda mediática esbozada por esta investigación indica que el PPdG es el partido que ocupa más noticias en los periódicos tradicionales. Las agendas políticas y mediáticas difieren en que esta última presta una singular atención a la estrategia tras las elecciones. Esta singular referencia a los pactos de gobierno no ha sido detectada en nuestro análisis, en contraposición a investigaciones recientes que identifican una importancia creciente de estos "meta-temas" (Rodríguez Díaz y Castromil, 2020).

En resumen, los resultados del estudio de las cuentas de Twitter, el debate electoral y los diarios de referencia aportan una serie de conclusiones sobre las agendas políticas y mediáticas para las elecciones gallegas de 2020. En primer lugar, los candidatos expusieron en Twitter una agenda temática fragmentada, con divergencias entre el candidato en el poder (Núñez Feijóo) y los pertenecientes a la oposición (Caballero y Pontón). Núñez Feijóo emitió tuits sobre aspectos programáticos como la economía y los servicios públicos. Por su parte, Caballero y Pontón priorizaron mensajes acerca de eventos políticos, el cambio y la movilización del voto. Su objetivo era promover variaciones en un sistema gallego proclive a las mayorías absolutas del PPdG.

Como segunda contribución, el tema con el que más interactúa la audiencia en Twitter en forma de de retuits es el Covid-19, seguido de los eventos políticos y la movilización del voto. Las dos últimas cuestiones eran fundamentales en la agenda temática de los líderes para esta red social, pero no así el coronavirus. Esto evidencia que no hay una transferencia exacta de relevancia, así como que la pandemia ha modificado los intereses de la ciudadanía.

En tercer lugar, el debate electoral televisivo giró en torno al Covid-19 y dos cuestiones programáticas como la economía y los servicios públicos. El tiempo dedicado a ellas fue similar entre los tres candidatos. Por tanto, no se observa una correlación directa entre la agenda temática expuesta en Twitter y la evidenciada en el debate de la Televisión de Galicia. El presidente y candidato Núñez Feijóo es el líder que denota una mayor similitud entre ambas.

Como última contribución, la agenda mediática ha versado sobre los eventos políticos y la estrategia postelectoral. La cuestión de los acuerdos postelectorales apenas se apreció en los medios seleccionados. Por su parte, los diarios de la muestra suelen focalizar sus piezas periodísticas en el PPdG o enfoques mixtos con varios partidos, con la excepción del Faro de Vigo, que articula una cobertura bastante homogénea en términos cuantitativos de las tres formaciones tradicionales.

El presente artículo tiene como limitación el tamaño reducido de la muestra, derivado del propio objeto de estudio, lo que obliga a implementar futuras investigaciones comparadas sobre comicios autonómicos para entender el papel del Covid-19 en las elecciones celebradas en 2020. Este estudio parte del interés por conocer la influencia de la agenda política a la mediática, estableciendo posibles correlaciones entre las agendas. Dichos procesos podrían también ser evaluados al revés. Aislar estos conjuntos de asuntos públicos resulta complejo, lo que espolea la necesidad de abordar la vertiente temática en varias manifestaciones discursivas que arrojen datos de interés ante un modelo de comunicación híbrido.

\section{Agradecimientos}

Este artículo ha sido traducido al inglés por Sophie Phillip. 


\section{Referencias bibliográficas}

Aleixandre-Benavent, R., Castelló-Cogollos, L. \& Valderrama-Zurián, J.-C. (2020). Información y comunicación durante los primeros meses de Covid-19. Infodemia, desinformación y papel de los profesionales de la información. Profesional de la información, 29(4), e290408. https://doi.org/10.3145/epi.2020.jul.08

Ardévol-Abreu, A., Gil de Zúñiga, H. \& McCombs, M. E. (2020). Orígenes y desarrollo de la teoría de la agenda setting en Comunicación. Tendencias en España (2014-2019). Profesional de la información, 29(4), e290414. https://doi.org/10.3145/epi.2020.jul.14 Ares, C. \& Rama, J. (2019). Las elecciones al Parlamento de Galicia (1981-2016): la importancia de la estrategia de transversalidad del PPdeG. En B. Gómez, L. Cabeza \& S. Alonso (eds.). En busca del poder territorial. Cuatro décadas de elecciones autonómicas en España (pp. 303-330). Madrid: Centro de Investigaciones Sociológicas.

Berrocal, S. (2011). Los debates electorales en televisión. Un estudio del caso español en las elecciones de 2008. En G. López García (ed.). Política binaria y spam electoral. Elecciones generales 2008: nuevas herramientas, viejas actitudes (pp. 147-168). Valencia: Tirant lo Blanch.

Brants, K., de Vreese, C., Möller, J. \& van Praag, P. (2009). The Real Spiral of Cynicism? Symbiosis and Mistrust between Politicians and Journalists. The International Journal of Press/Politics, 15(1), 25-40. https://www.doi.org/10.1177/1940161209351005

Campos-Domínguez, E. (2017). Twitter y la comunicación política. El profesional de la información, 26(5), 785-793. https://doi. org/10.3145/epi.2017.sep.01

Carballo, M., López-Escobar, E. \& McCombs, M. E. (2018). Communication, Public Opinion, and Democracy: New challenges. Communication \& Society, 31(4), 121-134. https://doi.org/10.15581/003.31.4.121-133

Casero-Ripollés, A. (2020). Impact of Covid-19 on the media system. Communicative and democratic consequences of news consumption during the outbreak. El profesional de la información, 29(2), e290223. https://doi.org/10.3145/epi.2020.mar.23

Casero-Ripollés, A., Alonso-Muñoz, L. \& Marcos-García, S. (2021). The Influence of Political Actors in the Digital Public Debate on Twitter About the Negotiations for the Formation of the Government in Spain. American Behavioral Scientist, 1-16. https:// doi.org/10.1177/00027642211003159

Castromil, A. R., Rodríguez-Díaz, R. \& Garrigós, P. (2020). La agenda política en las elecciones de abril de 2019 en España: programas electorales, visibilidad en Twitter y debates electorales. El profesional de la información, 29(2), e290217. https://doi. org/10.3145/epi.2020.mar.17

Cebrián, E., Vázquez, T. \& Olbarrieta, A. (2013). ¿Participación y democracia en los medios sociales?: El caso de Twitter en las elecciones vascas de 2012. AdComunica. Revista Científica de Estrategias, Tendencias e Innovación en Comunicación, 6, 39-63. https://doi.org/10.6035/132

Chavero-Ramírez, P., González, J. J., Bouza, F., Castromil, A. \& Rodríguez-Díaz, R. (2013). La mediatización de la agenda política: la discusión del pacto social como conflicto de agendas, 2009-2011. Revista Latina de Comunicación Social, 68, 639-655. https:// doi.org/10.4185/RLCS-2013-994 
Deltell, L., Claes, F. \& Osteso, J. M. (2013). Predicción de tendencia política por Twitter: Elecciones Andaluzas 2012. Ámbitos: Revista Internacional de Comunicación, 22, 1-13. https://bit.ly/2WpJMaQ

Fenoll, V., García-Ull, F. J. \& Rodríguez-Ballesteros, P. (2016). Valoración de candidatos en Twitter durante procesos electorales. El caso de las elecciones autonómicas valencianas de 2015. En Proceedings of the V Congreso Iberoamericano de Comunicación $A E-I C$, pp. 595-605. https://bit.ly/3F0ObST

Fernández Crespo, M. (2014). Metodología para el análisis de las redes sociales. En R. Cotarelo \& J. A. Olmeda (eds.). La democracia del siglo XXI. Política, medios de comunicación, internet y redes sociales (pp. 383-408). Madrid: Centro de Estudios Políticos y Constitucionales.

Gamir Ríos, J. (2016). Blogs, Facebook y Twitter en las Elecciones Generales de 2011. Estudio cuantitativo del uso de la web 2.0 por parte de los cabezas de lista del PP y del PSOE. Dígitos. Revista de Comunicación Digital, 2, 101-120. http://dx.doi.org/10.7203/ rd.v0i2.53

García Marín, J. (2015). La cobertura mediática de los debates electorales en España. Revista Española de Ciencia Política, 38, 135-161. https://bit.ly/2Z0bd9x

Gómez-Reino, M. (2009). El nacionalismo minoritario, de la marginalidad al gobierno: la trayectoria del Bloque Nacionalista Galego (1982-2007). Papers: Revista de Sociología, 92, 119-142. https://bit.ly/3ulVlMt

Guo, L. \& McCombs, M. (eds.) (2015). The Power of Information Networks: New Directions for Agenda Setting. Nueva York: Routledge.

Humanes, M. L. \& Moreno, M. A. (2012). El efecto agenda sobre los temas de campaña en las elecciones generales de 2008. AdComunica, 3, 191-207. http://dx.doi.org/10.6035/2174-0992.2012.3.12

Humanes, M. L. (2014). Exposición selectiva y partidismo de las audiencias en España. El consumo de información política durante las campañas electorales de 2008 y 2011. Palabra Clave, 17(3), 773-802. https://doi.org/10.5294/pacla.2014.17.3.9

Jungherr, A. (2016). Twitter use in election campaigns: A systematic literature review. Journal of Information Technology \& Politics, 13(1), 72-91. https://www.doi.org/10.1080/19331681.2015.1132401

Kim, Y., Kim, Yo. \& Zhou, S. (2017). Theoretical and methodological trends of agenda-setting theory. A thematic analysis of the last four decades of research. The Agenda Setting Journal, 1(1), 5-22. https://doi.org/10.1075/asj.1.1.03kim

Krippendorff, K. (2012). Content Analysis, An Introduction to Its Methodology. Thousand Oaks, CA: Sage Publications.

Labio, A. \& Pineda, A. (2016). Leftward Shift, Media Change? Ideology and Politics in Spanish Online-Only Newspapers After the 15-M Movement. International Journal of Communication, 10, 2661-2682. https://bit.ly/2ZJ4cZI

Lagares Díez, N., Jaráiz Gulías, E. \& Castro Martínez, P. (2020). La percepción de los debates como factor de decisión en el comportamiento electoral en las Elecciones Generales de abril de 2019. Revista Latina de Comunicación Social, 76, 39-58. https://doi. org/10.4185/RLCS-2020-1436

Larsson, A. O. \& Ihlen, Ø. (2015). Birds of a feather flock together? Party leaders on Twitter during the 2013 Norwegian elections. European Journal of Communication, 30(6), 666-681. https://doi.org/10.1177/0267323115595525 
López García, G., Gómez Nicolau, E. \& Torreblanca Devís, C. (2017). La construcción de la agenda en campaña electoral. En G. López García \& L. Valera Ordaz (coords.). Pantallas electorales el discurso de partidos, medios y ciudadanos en la campaña de 2015 (pp. 25-40). Barcelona: Editorial UOC.

López Meri, A. (2016). Twitter-retórica para captar votos en campaña electoral. El caso de las elecciones de Cataluña de 2015. Comunicación y Hombre, 12, 97-118. https://doi.org/10.32466/eufv-cyh.2016.12.190.97-118

López-Meri, A., Marcos-García, S. \& Casero-Ripollés, A. (2017). What do politicians do on Twitter? Functions and communication strategies in the Spanish electoral campaign of 2016. El profesional de la información, 26(5), 795-804. https://doi.org/10.3145/ epi.2017.sep.02

Masip, P., Aran-Ramspott, S., Ruiz-Caballero, C., Suau, J., Almenar, E. \& Puertas-Graell, D. (2020). Consumo informativo y cobertura mediática durante el confinamiento por el Covid-19: sobreinformación, sesgo ideológico y sensacionalismo. El profesional de la información, 29(3), e290312. https://doi.org/10.3145/epi.2020.may.12

Mazzoleni, G. (2010). La comunicación política. Madrid: Alianza Editorial.

McAllister, I. (2007). The personalization of politics. En R. Dalton \& H. D. Klingemann (eds.). The Oxford Handbook of Political Behaviour (pp. 571-588). Oxford: Oxford University Press.

McCombs, M. E. \& Shaw, D. (1972). The agenda-setting function of mass media. Public Opinion Quarterly, 36(2), 176-187. https:// www.doi.org/10.1086/267990

McCombs, M. E. (2006). Estableciendo la agenda. El impacto de los medios en la opinión pública y en el conocimiento. Barcelona: Paidós

Parmelee, J. H. \& Bichard, S. L. (2012). Politics and the Twitter revolution. How tweets influence the relationship between political leaders and the public. Lanham, MD: Lexington Books.

Patterson, T. E. (1980). The Mass Media Election. Hour the Americans Choose their President. Nueva York: Praeger.

Pérez-Curiel, C., Rivas-de-Roca, R. \& García-Gordillo, M. (2021). Impact of Trump's Digital Rhetoric on the US Elections: A View from Worldwide Far-Right Populism. Social Sciences, 10(5), 152. https://doi.org/10.3390/socsci10050152

Rivas-de-Roca, R., García-Gordillo, M. \& Rojas-Torrijos, J. L. (2021). Estrategias comunicativas en Twitter y portales institucionales durante la segunda ola de Covid-19: análisis de los gobiernos de Alemania, España, Portugal y Reino Unido. Revista Latina de Comunicación Social, 79, 49-72. https://doi.org/10.4185/RLCS-2021-1517

Rodríguez-Díaz, R. \& Castromil, A. R. (2020). Elecciones 2015 y 2016 en España: el debate desde los temas a los “meta-temas" de agenda. Revista Latina de Comunicación Social, 76, 209-227. https://doi.org/10.4185/RLCS-2020-1444

Rodríguez-Virgili, J., López-Escobar, E. \& Tolsa, A. (2011). La percepción pública de los políticos, los partidos y la política, y uso de medios de comunicación. Comunicación y Sociedad, 24(2), 7-40. https://bit.ly/3CYMT9j

Rúas-Araújo, J., Pérez-Curiel, C. \& López-López, P. C. (2020). New Challenges and Threats for Journalism in the Post-truth Era: Fact-checking and the Fake News Combat. En C. Toural-Bran, A. Vizoso, S. Pérez-Seijo, M. Rodríguez-Castro \& M. C. Negreira-Rey (eds.). Information Visualization in the Era of Innovative Journalism (pp. 154-160). NuevaYork/Londres: Routledge. 
Sádaba Rodríguez, I. (2012). Introducción a la investigación social online. En M. Arroyo Menéndez \& I. Sádaba Rodríguez (coords.). Metodología de la investigación social: técnicas innovadoras y sus aplicaciones (pp. 197-216). Madrid: Síntesis.

Strömbäck, J. (2008). Four phases of mediatization: An analysis of the mediatization of politics. The International Journal of Press/ Politics, 13(3), 228-246. https://doi.org/10.1177/1940161208319097

Stromer-Galley, J. (2014). Presidential Campaigning in the Internet Age. Oxford: Oxford University Press.

Tenscher, J., Koc-Michalska, K., Lilleker, D. G., Mykkänen, J., Walter, A. S., Findor, A., Jalali, C. \& Róka, J. (2016). The professionals speak: Practitioners' perspectives on professional election campaigning. European Journal of Communication, 3(2), 95-119. https://doi.org//10.1177/0267323115612212

Vaccari, C., Chadwick, A. \& O'Loughlin, B. (2015). Dual screening the political: Media events, social media, and citizen engagement. Journal of Communication, 65(6), 1041-1061. https://doi.org/10.1111/jcom.12187

Valera Ordaz, L. (2015). Building the news media agenda: Spanish political parties' influence on traditional media and blogosphere agendas during 2011 electoral campaign. Communication \& Society, 28(3), 115-134. https://doi.org/10.15581/003.28.3.115-134

Wagner, M. (2016). Selective exposure, information utility, and the decision to watch televised debates. International Journal of Public Opinion Research, 29(4), 533-553. https://doi.org/10.1093/ijpor/edw016

Warf, B. \&. Ferras, C. (2015). Nationalism, identity and landscape in contemporary Galicia. Space and Polity, 19(3), p256-272. https://doi.org/10.1080/13562576.2015.1080425

Zugasti Azagra, R. \& García Ortega, C. (2018). Los temas de los líderes políticos españoles en Twitter. Análisis de las dos campañas electorales de 2015. Revista ICONO 14. Revista Científica de Comunicación y Tecnologías Emergentes, 16(1), 136-159. https://doi. org/10.7195/ri14.v16i1.1137 
